This item was submitted to Loughborough's Research Repository by the author.

Items in Figshare are protected by copyright, with all rights reserved, unless otherwise indicated.

\title{
An optimum sensor selection design framework applied to an electro- magnetic suspension system
}

PLEASE CITE THE PUBLISHED VERSION

http://dx.doi.org/10.1109/SYSTOL.2010.5676004

PUBLISHER

(C) IEEE

VERSION

VoR (Version of Record)

LICENCE

CC BY-NC-ND 4.0

\section{REPOSITORY RECORD}

Michail, Konstantinos, Argyrios C. Zolotas, and Roger M. Goodall. 2019. "An Optimum Sensor Selection Design Framework Applied to an Electro-magnetic Suspension System”. figshare.

https://hdl.handle.net/2134/9257. 
This item was submitted to Loughborough's Institutional Repository (https://dspace.lboro.ac.uk/) by the author and is made available under the following Creative Commons Licence conditions.

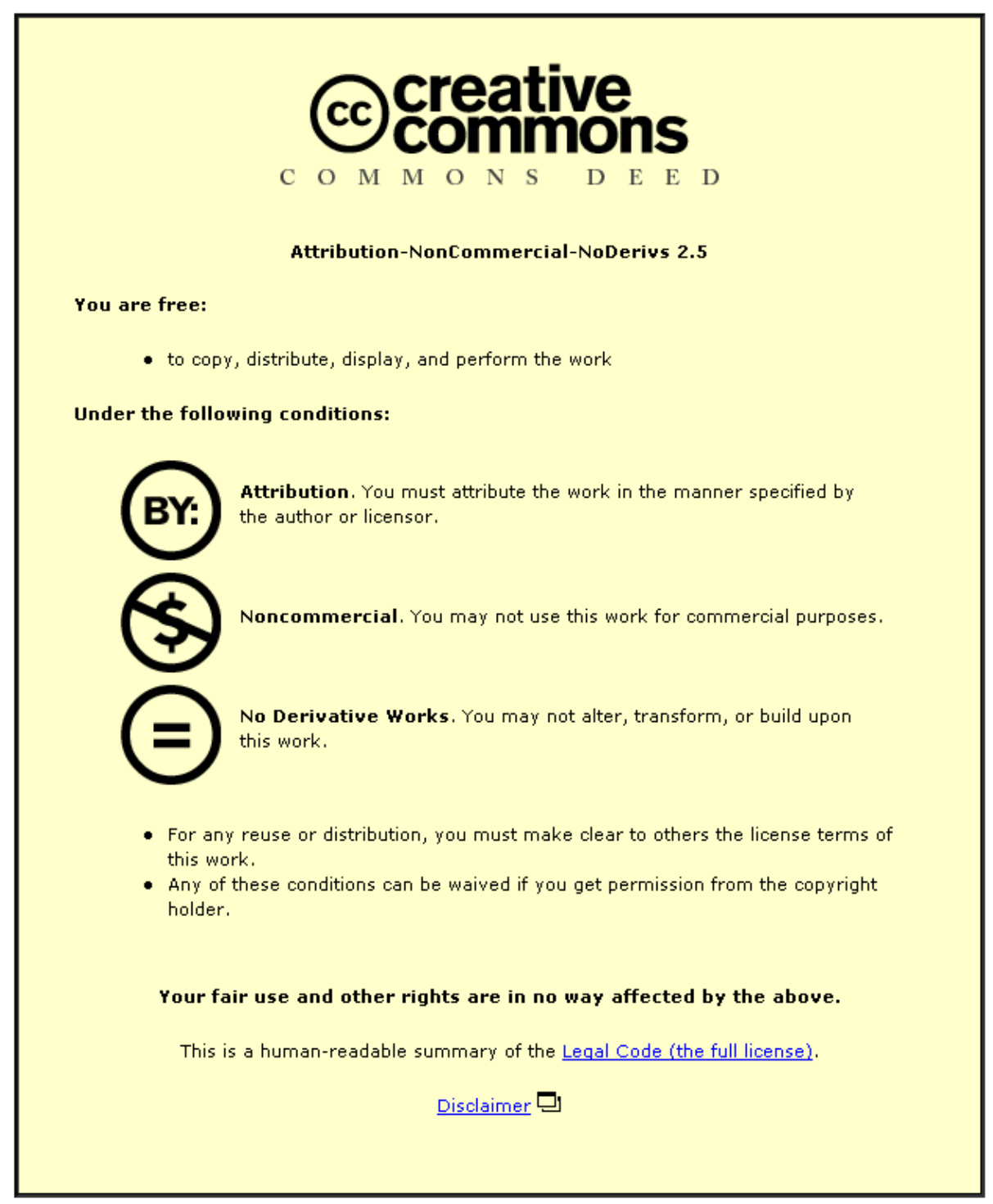

For the full text of this licence, please go to: http://creativecommons.org/licenses/by-nc-nd/2.5/ 


\title{
An Optimum Sensor Selection Design Framework Applied to an Electro-Magnetic Suspension System
}

\author{
Konstantinos Michail Argyrios Zolotas and Roger Goodall
}

\begin{abstract}
This paper presents a systematic design framework for selecting the best sensor set to control an EMS system subject to satisfying a set of complex requirements such as optimum performance, robustness and sensor fault tolerance. The design framework combines the $\mathcal{H}_{\infty}$ robust control design, fault tolerance concept and heuristic approaches. The efficacy of the scheme is illustrated via application to an EMS system.
\end{abstract}

\section{INTRODUCTION}

A common problem in control design is the selection of sensors subject to given set of reliability requirements. The complexity of the problem increases with increasing number of sensors and also that of the reliability set of requirements to achieve. In this context, a selection scheme is proposed to ease design on choice of best sensor sets subject to given design constraints. In fact, the selection of sensors subject to optimum performance, robustness, minimum number of sensors and sensor fault tolerance is a rather complex and often impossible to do manually especially if there are a lot of sensor sets to select from. Under this scope the authors present a novel systematic framework that is able to identify the best sensor set subject to aforementioned properties. The proposed framework combines $\mathcal{H}_{\infty}$ robust control, fault tolerance and heuristic optimisation. In particular, the scheme targets a specific EMS control design problem with a set of realistic constraints in terms of suspension design. The EMS system is a non-linear, unstable, safety-critical system with non-trivial requirements [8], [9]. Such type of suspension is using an electromagnetic force that attracts the vehicle onto the track avoiding use of wheels on rail (i.e. mechanical contact is avoided). Previous work presented in [4] $\mathcal{H}_{\infty}$ control of EMS system but without considering the sensor selection problem. In [15] the optimum sensor selection has been considered but excluding sensor Fault tolerance and non-linearities of the EMS system. Other modern control methods has been used in combination with the same optimisation framework include Linear Quadratic Gaussian control in [14], [16] and Loop Shaping Design in [17].

The paper is organised as follows: Section II presents the EMS system model including non-linearities and linearised state space model, as well as the requirements of the suspension are given including the objective functions and the design limitations for the closed-loop response. Section III

This work was supported in part under the EPSRC (UK) project Grand Ref. EP/D063965/1 and BAE Systems (SEIC), UK.

K. Michail, A. Zolotas, R. Goodall are with the Department of Electrical and Electronic Engineering, Loughborough University, Loughborough, LE11 3TU, UK. kon_michael@ieee.org, $\{$ a.c.zolotas,r.m.goodal1\}@lboro.ac.uk presents the proposed design framework along with MultiObjective $\left(\mathcal{M} . \mathcal{O}\right.$.) $\mathcal{H}_{\infty}$ robust control design for the EMS system and the M.O. constrained optimisation via evolutionary algorithm that is important for the optimisation of the closed-loop performance. Section IV gives simulations and discussion of results while conclusions and some future work are given Section V.

\section{EMS MODELLING AND REQUIREMENTS}

\section{A. The EMS Model}

The 1DOF (degree-of-freedom) model represents the quarter of a typical MAGLEV vehicle. In this section the single degree of freedom model of an Electro-Magnetic suspension (EMS) system is analyzed. The basic quarter car model of the MAGLEV vehicle is shown in Fig. 1. Note that this is a single-stage electro-magnetic suspension that has been shown to be suitable for low speed vehicles [9]. As it can be seen the suspension consists of an electromagnet with a ferromagnetic core and a coil of $N_{c}$ turns which is attracted to the rail that is made of ferromagnetic material. The carriage mass $\left(M_{s}\right)$ is attached on the electromagnet, with $z_{t}$ the rail's position and $z$ the electromagnet's position. The air gap $\left(z_{t}-z\right)$ is to be maintained close to the operating condition required. There are four important variables in an

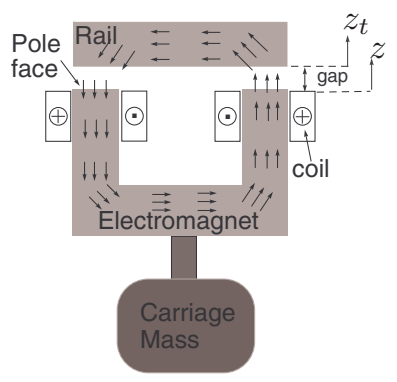

Fig. 1. Single-stage suspension for MAGLEV vehicles.

electromagnet named as force F, flux density B, air gap G and the coil's current I that give non-linear characteristics to the suspension as described in [10], [11].

Assuming that the motion vertically downwards is taken as positive the non-linear model of the EMS system is described by Newton's equation of motion in (1) and the voltage $V_{c}$ in (2) across the electromagnet's coil from Kirchoff's law. Equations (3) and (4) describe the force and flux density and 
the air gap velocity respectively [10].

$$
\begin{aligned}
& M_{s} \frac{d^{2} Z}{d t^{2}}=M_{s} g-F \\
& V_{c}=I R_{c}+L_{c} \frac{d I}{d t}+N_{c} A_{p} \frac{d B}{d t} \\
& B=K_{b} \frac{I}{G}, F=K_{f} B^{2} \\
& \frac{d G}{d t}=\frac{d z_{t}}{d t}-\frac{d Z}{d t}
\end{aligned}
$$

where $g$ is the gravity acceleration constant which is $9.81 \mathrm{~m} / \mathrm{s}^{2}$. The linearisation of the non-linear MAGLEV suspension model is based on small perturbations around the operating point. The following definitions are used with lower case letters defining the small variation around the operating point and subscript 'o' referring to the operating point.

$$
\begin{aligned}
& B=B_{o}+b, F=F_{o}+f \\
& I=I_{o}+i, G=G_{o}+\left(z_{t}-z\right) \\
& V_{c}=V_{o}+u_{c}, Z=Z_{o}+z
\end{aligned}
$$

Following the linearization procedure in [10] the state space description of the EMS system can be expressed in state space form as in (8) where the selected states are $x=$ $\left[\begin{array}{lll}i & \dot{z} & \left(z_{t}-z\right)\end{array}\right]^{T}$ and the output equation corresponds to the following five measurements: $i$, the coil's current, $b$ the flux density, $\left(z_{t}-z\right)$ is the air gap, $\dot{z}$ the vertical velocity and $\ddot{z}$ the vertical acceleration. The matrices $A, B_{u_{c}}, B_{\dot{z}_{t}}$ and $C$ are given by (9)-(11).

$$
\begin{aligned}
& \dot{x}=A x+B_{u_{c}} u_{c}+B_{\dot{z}_{t}} \dot{z}_{t} \\
& y=C x \\
& A=\left[\begin{array}{ccc}
-\frac{R_{c}}{L_{c}+\frac{K_{b} N_{c} A_{p}}{G_{o}}} & -\frac{K_{b} N_{c} A_{p} I_{o}}{G_{o}^{2}\left(L_{c}+\frac{K_{b} N_{c} A_{p}}{G_{o}}\right)} & 0 \\
-2 K_{f} \frac{I_{o}}{M_{s} G_{o}^{2}} & 0 & 2 K_{f} \frac{I_{o}^{2}}{M_{s} G_{o}^{3}} \\
0 & -1 & 0
\end{array}\right] \\
& B_{u_{c}}=\left[\begin{array}{c}
\frac{1}{L_{c}+\frac{K_{b} N_{c} A_{p}}{G_{o}}} \\
0
\end{array}\right], B_{\dot{z}_{t}}=\left[\begin{array}{c}
\frac{K_{b} N_{c} A_{p} I_{o}}{G_{o}^{2}\left(L_{c}+\frac{K_{b} N_{c} A_{p}}{G_{o}}\right)} \\
0 \\
1
\end{array}\right] \\
& C=\left[\begin{array}{ccc}
1 & 0 & 0 \\
\frac{K_{b}}{G_{o}} & 0 & -\frac{K_{b} I_{o}}{G_{o}^{2}} \\
0 & 0 & 1 \\
0 & 1 & 0 \\
-2 K_{f} \frac{I_{o}}{M_{s} G_{o}^{2}} & 0 & 2 K_{f} \frac{I_{o}^{2}}{M_{s} G_{o}^{3}}
\end{array}\right]
\end{aligned}
$$

The sensor combinations can be obtained by using the output matrix $\mathrm{C}$ in (11). The total number of sensor sets, $N_{s}$ is given as $N_{s}=2^{n_{s}}-1$. Where $n_{s}$ is the total number of sensors. Given that the EMS system has 5 outputs there are 31 candidate sensor sets.

The electromagnet design of MAGLEV vehicles is described in more details in [11]. A typical quarter car vehicle of
$1000 \mathrm{~kg}$ requires an operating force of $F_{o}=M_{s} \times g$. The operating air gap $\left(G_{o}\right)$ is at $15 \mathrm{~mm}$ to accommodate the track roughness. According these requirements the rest of the parameters can be calculated as shown on Table I.

TABLE I

\begin{tabular}{llll}
\multicolumn{4}{c}{ PARAMETERS OF THE EMS SYSTEM. } \\
\hline \hline \multicolumn{1}{c}{ Parameter } & \multicolumn{1}{c}{ Value } & \multicolumn{1}{c}{ Parameter } & \multicolumn{1}{c}{ Value } \\
\hline Op. air gap, $G_{o}$ & $0.015 \mathrm{~m}$ & Carriage Mass, $M_{s}$ & $1000 \mathrm{~kg}$ \\
Op. flux density, $B_{O}$ & $1 \mathrm{~T}$ & Coil's Resistance, $R_{c}$ & $10 \Omega$ \\
Op. current, $I_{O}$ & $10 \mathrm{~A}$ & Coil's Inductance, $L_{c}$ & $0.1 \mathrm{H}$ \\
Op. voltage, $V_{o}$ & $100 \mathrm{~V}$ & Number of turns, $N_{c}$ & 2000 \\
Op. force, $F_{o}$ & $9810 \mathrm{~N}$ & Pole face area, $A_{p}$ & $0.01 \mathrm{~m}^{2}$ \\
\hline \hline
\end{tabular}

Note: Op. stands for Operating.

\section{B. DESIGN REQUIREMENTS AND INPUTS TO THE EMS}

1) Stochastic Inputs: The stochastic inputs are random variations of the rail position as the vehicle moves along the track. This is caused by the steel rail installation discrepancies due to track-laying inaccuracies and unevenness. Considering the vertical direction, the velocity variations can be approximated by a double-sided power spectrum density (PSD) expressed as

$$
S_{\dot{z}_{t}}=\pi A_{r} V_{v}
$$

where $V_{v}$ is the vehicle speed (taken as $15 \mathrm{~m} / \mathrm{s}$ in this case) and $A_{r}$ represents the roughness and it is assigned a value as $1 \times 10^{-7}$ for high quality track. Then the corresponding autocorrelation function is given as:

$$
R(\tau)=2 \pi^{2} A_{r} V_{v} \delta(\tau)
$$

Regardless a linear controller is used, the simulations are actually based on the implementation to the nonlinear model. Hence, we calculate the RMS values of the required quantities (acceleration, current etc) using time history data. Details of implementation of linear controller onto a nonlinear model can be found in [7].

2) Deterministic Input: The main deterministic input to the suspension in the vertical direction is due to the transition onto a gradient. In this work, the deterministic input (see Fig. 2) is a gradient of $5 \%$ at a vehicle speed of $15 \mathrm{~m} / \mathrm{s}$, an acceleration of $0.5 \mathrm{~m} / \mathrm{s}^{2}$ and a jerk of $1 \mathrm{~m} / \mathrm{s}^{3}$.

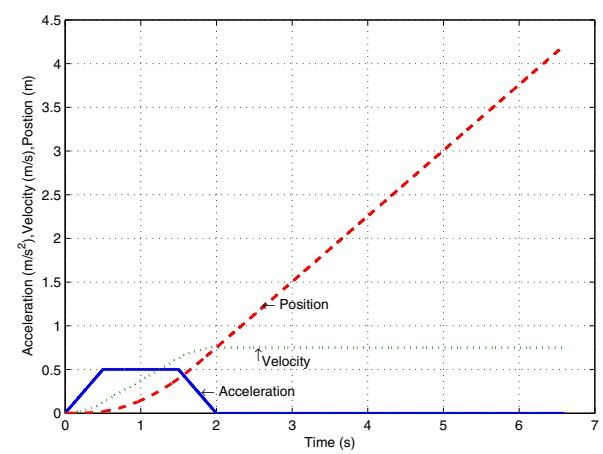

Fig. 2. Deterministic input to the suspension with a vehicle speed of $15 \mathrm{~ms}^{-1}$ and $5 \%$ gradient. 
3) EMS Control Properties: The design requirements for an electromagnetic suspension (EMS) depend on the type and speed of the train. The dynamic characteristics of a MAGLEV suspension are well described in [8], [9]. It is focused upon the low speed Birmingham Airport Maglev vehicle EMS suspension requirements which has operated successfully in the UK for a period of 12 years in the 1980s and 1990s. Fundamentally, there is a trade-off between the deterministic and stochastic responses of the EMS system. Table II tabulates the design limitations for the deterministic and stochastic features. The deterministic features are limited to the maximum standard values. The stochastic ones are set as objectives to be minimized i.e. the vertical acceleration $\ddot{z}_{r m s}$ (improve ride quality) and the RMS current variations $i_{r m s}$ from the stochastic response. The robustness margin $\gamma_{\text {opt }}$ from the $\mathcal{H}_{\infty}$ controller design and the noise amplitude on the control effort $u_{n_{r m s}}$ when noisy measurements as also minimized. Hence, the objective functions $\phi_{i}$ to be minimized are formally written as:

$$
\phi_{1}=i_{r m s}, \phi_{2}=\gamma_{o p t}, \phi_{3}=\ddot{z}_{r m s}, \phi_{4}=u_{n_{r m s}}
$$

\section{TABLE II}

CONSTRAINTS ON THE EMS SYSTEM PERFORMANCE.

\begin{tabular}{l|l}
\hline \hline \multicolumn{1}{c|}{ EMS limitations } & \multicolumn{1}{c}{ Value } \\
\hline RMS acceleration $\simeq 5 \%^{\prime} g^{\prime}, \ddot{z}_{r m s}$ & $\leq 0.5 \mathrm{~ms}^{-2}$ \\
RMS air gap variation, $\left(z_{t}-z\right)_{r m s}$ & $\leq 5 \mathrm{~mm}$ \\
RMS control effort, $u_{c_{r m s}}$ & $\leq 300 \mathrm{~V}\left(3 I_{0} R_{c}\right)$ \\
Maximum air gap deviation, $\left(z_{t}-z\right)_{p}$ & $\leq 7.5 \mathrm{~mm}$ \\
Control effort, $u_{c_{p}}$ & $\leq 300 \mathrm{~V}\left(3 I_{0} R_{c}\right)$ \\
Settling time, $t_{s}$ & $\leq 3 \mathrm{~s}$ \\
Air gap Steady state error, $\left(z_{t}-z\right)_{e_{s s}}$ & $=0$ \\
\hline \hline
\end{tabular}

\section{THE SENSOR OPTIMISATION FRAMEWORK}

The proposed framework can be summarised in the flow chart of Fig. 3. The particular points include the use of $\mathcal{H}_{\infty}$ robust control design, the heuristic optimisation (evolutionary algorithms) method for tuning the controller subject to strict requirements (objectives and constraints) for each feasible sensor set of the EMS system.

Prior to running the algorithm, some parameters are assigned including evolutionary algorithms parameters, controller selection criteria, $f_{c_{i}}$, and the user's controller selection criterion, $f_{k} . f_{c_{i}}$ and $f_{k}$ make sure that the selected controller results in a desired closed-loop performance. Starting the optimisation procedure, the first sensor set is selected and the evolutionary algorithm seeks the optimum Pareto front of the objective functions in (14) (i.e. the trade-off between the objective functions) subject to the constraints listed in Table II. In the sequence, the $\mathcal{H}_{\infty}$ designed controllers satisfying all constraints are selected based on overall constraint violation function, $\Omega$ (see (18) in Section III-C). If there is no sufficient controller then the controller which results to minimum $\Omega$ is selected and the optimisation proceeds to the next sensor set. Otherwise, those controllers satisfying $\Omega$ are selected. The next step is to select those controllers that satisfy the controller selection criteria $f_{c_{i}}$ and finally, the user's controller selection criteria, $f_{k}$ is used to select the controller which results in the desired closed-loop response. The optimally tuned controller is saved and the algorithm moves to the next feasible sensor set until all feasible sensor sets are checked.

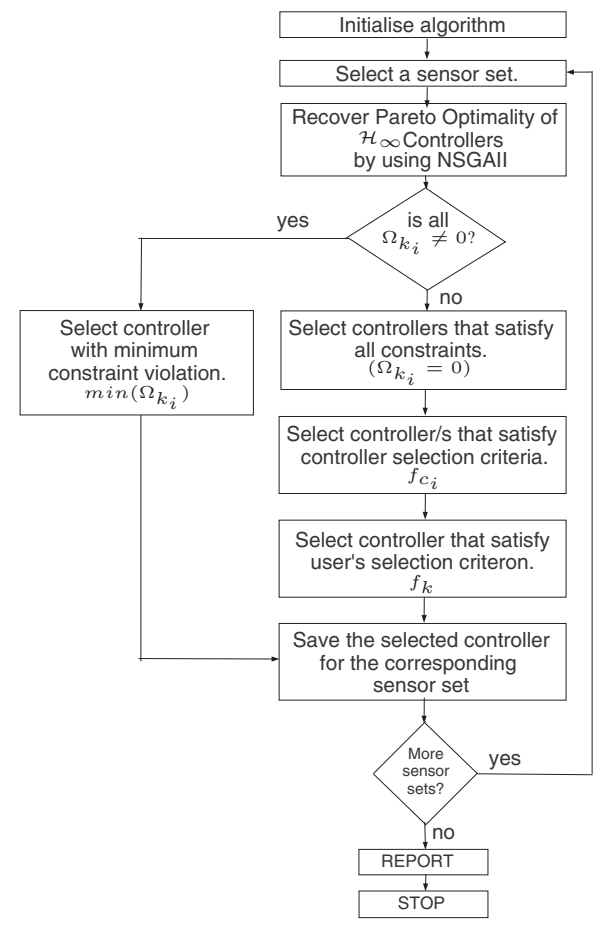

Fig. 3. Flow chart of the sensor optimisation framework with robust $\mathcal{H}_{\infty}$ control design.

\section{A. M.O. $\mathcal{H}_{\infty}$ Robust Control for the EMS}

The M.O. $\mathcal{H}_{\infty}$ Robust control design concept has been very well described in [19]. The aim is to design a controller that the disturbances mentioned in Section II-B are sufficiently rejected. The problem setup in the context of sensor optimisation is illustrated in Fig. 4 where the EMS system State Space model in (8) is transformed into a generalised form in (15).

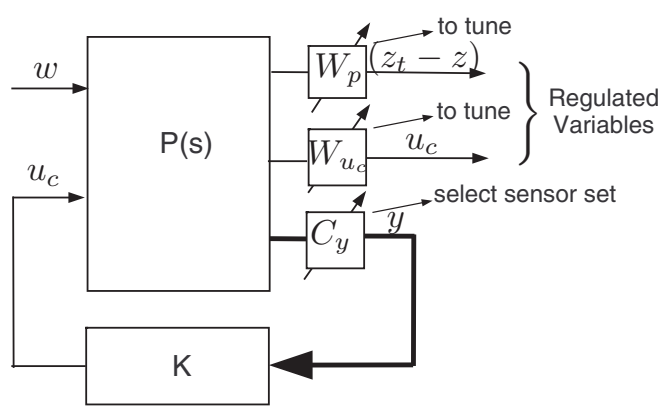

Fig. 4. M.O. generalised plant configuration for the EMS system. 


$$
\begin{aligned}
& \dot{x}=A x+B_{\dot{z}_{t}} w+B_{u} u \\
& z_{\infty}=C_{\infty} x+D_{\infty 1} w+D_{\infty 2} u \\
& y=C_{y} x+D_{y 1} w+D_{y 2} u
\end{aligned}
$$

where $w$ are exogenous inputs (deterministic and stochastic as described in Section II-B.3), $u$ is the EMS input, $z_{\infty}$ is the regulated outputs (i.e., $u_{c}$ is the control effort, $\left(z_{t}-z\right)$ is the air gap) and $y$ is the corresponding sensor set. Each sensor set is selected by manipulating the output matrix $\left(C_{y}\right)$.

The controller is designed in such a way that the infinity norm of the closed-loop transfer function from the exogenous inputs to the regulated outputs is minimized subject to the EMS requirements mentioned in Section II-B.3:

$$
\left\|T_{z_{\infty} w}\right\|_{\infty}<\gamma_{o p t}
$$

Note the controller is branded stabilising, thus if necessary another check of controller stability itself might be added if necessary. Unstable controllers are not favourable when they are to be used in switching schemes therefore the algorithm rejects all unstable stabilizing controllers. For each selected sensor set, (16) is solved for each random pair of weighting functions that is produced by the genetic algorithm via Linear Matrix Inequalities. This can be performed in MATLAB environment using function 'hinfmix' (robust control toolbox). The weighting filters $W_{p}$ and $W_{u_{c}}$ are appropriately selected low pass and high pass filters (17) to adjust the performance of the controller with parameters tuned via evolutionary algorithms. There is no generic procedure to select weighting filters as this can be classified as application dependent. However, some guidelines on selecting the weights for $H_{\infty}$ design of a plant are suggested in [19], and these are followed here for simplicity.

$$
W_{p}=\left(\frac{\frac{s}{M_{p}^{1 / n_{p}}}+\omega_{b}}{s+\omega_{b} A_{p}^{1 / n_{p}}}\right)^{n_{p}} \quad W_{u_{c}}=\left(\frac{\tau s+A_{u}^{1 / n_{u}}}{\frac{\tau}{M_{u}^{1 / n_{u}}} s+1}\right)^{n_{u}}
$$

In the performance weighting $\left(W_{p}\right), M_{p}$ is the high frequency gain, $A_{p}$ the low frequency gain and $\omega_{b}$ the crossover frequency. For the control effort weight $\left(W_{u_{c}}\right), \tau$ determines the crossover frequency, $A_{u}$ is the low frequency gain and $M_{u}$ is the high frequency gain. Both $n_{p}$ and $n_{u}$ control the roll-off rates of the filters, equal to 1 in this case i.e. first order filters. The controller output is fixed, as this is only the applied voltage to the EMS system. The controller inputs, however, vary based upon the utilised sensor set. i.e., SISO controller for 1 sensor; MISO controllers for more sensor combinations. Moreover, the order of the controller is fixed to the order of the plant plus the order of the chosen filters i.e. $3+2=5^{t h}$ order controller. Although the order of the controller is low if higher order controllers are necessary then controller reduction techniques can be easily adopted to the proposed framework.

\section{B. Sensor Fault Tolerance Scheme}

Fault tolerance is a subject that has been a main point of research studies in the last years [1], [2]. In this paper the aim is to recover the stability and performance in an optimum manner under multiple sensor failures. Under this scope the Active Fault Tolerant Control (AFTC) concept is used [18], [20]. The AFTC concept is composed from a bank of $\mathcal{H}_{\infty}$ designed controllers. When multiple sensor faults happen remedial actions are taken by controller reconfiguration. The recovery of the performance is aimed by using the remaining healthy sensors (sub-set of the selected sensor set) as depicted in Fig. 5. The reconfiguration scheme requires a Fault Detection and Isolation mechanism in order to detect and isolate the faulty sensors while it produces the controller reconfiguration signal. Note that the switching delay is assumed to be negligible for simplicity. Typically, to detect a fault a common way is to monitor the residual of two signals. The residuals for each output is typically produced by using dedicated observers [12]. A bank of

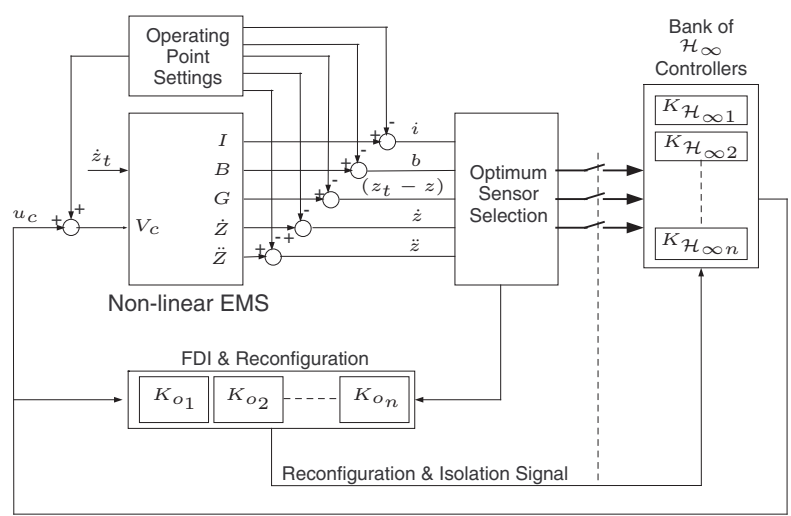

Fig. 5. AFTC diagram for multiple sensor failures using a bank of $\mathcal{H}_{\infty}$ designed controllers.

dedicated observers (i.e. $K_{o_{1}}, K_{O_{2}} \ldots K_{o_{n}}$ ) is used to monitor the condition of each sensor as depicted in Fig. 5. Isolating the faulty sensors is done by taking the sensor out of the circuit in such a way that the faulty signal is not fed to the new controller (i.e. switching). Sensor faults modelling can be done in three ways [12]: (i) abrupt fault (stepwise) (i)incipient fault (drift-like) and (iii)intermittent fault. In this paper the first sensor fault model is considered.

\section{Multi-objective Constrained Optimisation}

Heuristic approaches are very powerful optimisation tools that are implemented in many engineering problems and particularly evolutionary algorithms have been extensively implemented in control engineering [6]. Different types of genetic algorithms have been developed the last years and they are well summarized in [13]. In this research work, the recently developed genetic algorithm based on nondominated sorting of the population, Non-Dominated Sorting Genetic Algorithm II (NSGA-II) is used that proofs to be a powerful optimization tool. Details on NSGA-II can be found in [5] for the interested reader. NSGA-II is an evolutionary process that requires some parameters to be assigned in order to ensure proper population convergence towards optimum Pareto front. These are mainly selected from experience rather than from a-priori knowledge of the 
optimisation problem. The crossover probability is generally selected to be large in order to have a good mix of genetic material. The crossover probability is set to $90 \%$ and the mutation probability is defined as $1 / n_{u}$ where, $n_{u}$ is the number of variables. The population to be used consists of 50 chromosomes and the stopping criterion is the maximum generation number set at 200 .

To achieve the desired closed-loop response a constraint handling technique is necessary. Constraint handling methods with genetic algorithms can be done differently [3]. The dynamically updated penalty function approach is used to achieve the constraint within limits. A rigorous description of this method for the proposed systematic framework is described in [15]. This method is using a function in order to 'guide' the objective functions in (14) towards solution area where the desired constraints on Table II are satisfied. The overall constraint violation function is given as

$$
\Omega\left(k^{(j)}, f^{(i)}\right)=\sum_{j=1}^{J} \omega_{j}\left(k^{(j)}\right)+\sum_{i=1}^{I} \psi_{i}\left(f^{(i)}\right)
$$

where, $\omega_{j}$ is the $j^{\text {th }}$ soft constraint violation for the corresponding $j^{\text {th }}$ quantity to be constrained $(k)$ and $J$ is the total number of soft constraints. Similarly, $\psi$ is the hard constraint violation for the $i^{\text {th }}$ quantity to be constrained $(f)$. The overall constraint violation function serves as a controller selection criterion within the systematic framework as described at the beginning of this section.

\section{SIMULATION AND DATA ANALYSIS}

The overall algorithm is tested in MATLAB R2009b simulation environment without Java function due to large computational need (simulation based). The computer used is the powerful DELL T610 with $2.93 \mathrm{GHz}$ Intel@Xeon@X5570 processor and 8GB RAM.The average simulation time per sensor set was about 6 hours and the procedure for all feasible sensor sets takes around 152 hours.

The controller selection criteria $\left(f_{c_{i}}, f_{k}\right)$ for the desired closed-loop response are given as follows

$$
\begin{aligned}
& f_{c_{1}} \equiv \ddot{z}_{r m s} \leq 0.5 m / s^{2}, f_{c_{2}} \equiv \gamma_{o p t} \leq 1, \\
& f_{k} \equiv \min \left(u_{c_{r m s}}\right)
\end{aligned}
$$

From the simulation results it was found that the proposed systematic framework is able to identify, stable, stabilizing controllers for 24/31 sensor sets while four of them do not satisfy $\Omega$ in (18). However, they could be used if the constraint violation is not critical for the (perhaps degraded) performance of the suspension.

Table III lists some sensor sets selected for a deeper analysis of the results. The second column is the sensor sets and the first the corresponding identification number. The next four columns are the variables from the stochastic closed loop response while the further four shows the variable values from the deterministic response. The next column is the resulting robustness margin from the $\mathcal{H}_{\infty}$ robust control design and the $12^{t h}$ column lists the resulting RMS level of the noise on the input voltage. The $13^{\text {th }}$ column shows whether the overall constraint violation function, $\Omega$ is satisfied or not. The last column is the Sensor Fault Accommodation Ratio (SFAR). The SFAR is the ratio of the total number of sensor fault conditions that can be accommodated by using sub-sets of the main sensor set and the total sensor fault combinations. Obviously, single measurements (id:1-5) cannot be used neither for control nor for sensor fault tolerance control. Moreover, id: 1 and id:3 cannot satisfy $\Omega$ and on the other hand id:2,3 and 5 do not include stable stabilizing controller (in fact, single measurements could be used but in this case with unstable stabilising controller, see [15]). Proceeding to double measurements (e.g. id:6 and id:7) it can be seen that control is possible but the SFAR is zero for all of them. Moreover id:8 with three measurements has a SFAR of 2/7. Adding more sensors SFAR increases even if the sensor fault combinations number increases as well. Note that by using id:10 the SFAR is $8 / 15$ while moving to the full sensor set (id:13) the SFAR is similar. To increase the SFAR the id:1 and id:3 could be considered. Inspecting the related it can be seen that there are some constraint violation on the vertical acceleration, settling time and steady state error but not as critical. Thus, they can be used for sensor fault tolerance even with slightly degraded performance. In this context, id:8 can be used which has SFAR 4/7. However, extra care should be taken to protect the suspension under current and air gap measurements fault condition (i.e. $b$ remains alone). In that case, either current and/or air gap should be considered for triple redundancy for highest protection.

Figure 6 illustrates the faulty profile of current measurement, from $1 \mathrm{sec}$ onwards. However, the fault is accommodated as seen from Fig. 7 on air gap response.

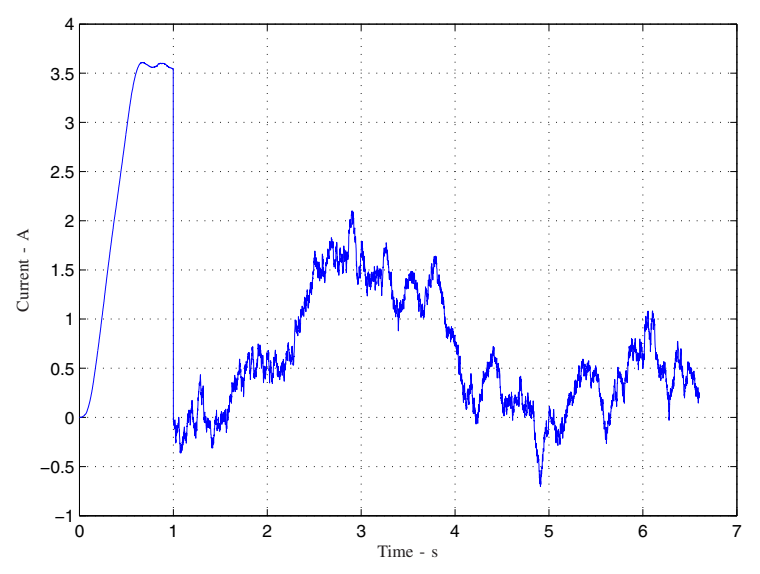

Fig. 6. Current fault profile.

\section{CONCLUSION AND FUTURE WORKS}

\section{A. Conclusion}

The proposed design framework is able to simplify the sensor selection procedure in an optimum manner subject to complex requirements. It combines the $\mathcal{H}_{\infty}$ robust control and optimisation methods with its efficacy illustrated on the EMS system. 
TABLE III

OPTIMISED SENSOR CONFIGURATIONS WITH $\mathcal{H}_{\infty}$ ROBUST CONTROL DESIGN.

\begin{tabular}{|c|c|c|c|c|c|c|c|c|c|c|c|c|c|}
\hline \multirow[b]{3}{*}{ id } & \multirow[b]{3}{*}{ Sensor set } & \multicolumn{4}{|c|}{ Stochastic input response } & \multicolumn{4}{|c|}{ Deterministic input response } & \multirow[b]{2}{*}{$\gamma$} & \multirow[b]{2}{*}{$u_{n_{r m s}}$} & \multirow[b]{2}{*}{$\Omega$} & \multirow[b]{2}{*}{$S F A R$} \\
\hline & & $g_{r m s}$ & $u_{c_{r m s}}$ & $\ddot{z}_{r m s}$ & $i_{r m s}$ & $g_{p}$ & $u_{c_{p}}$ & $t_{s}$ & $e_{s s}$ & & & & \\
\hline & & $\mathrm{mm}$ & $\mathrm{V}$ & $m s^{-2}$ & $A$ & $\mathrm{~mm}$ & $\mathrm{~V}$ & $s$ & & & $\mathrm{~V}$ & & \\
\hline 1 & $i$ & 2.79 & 32.35 & 0.73 & 2.19 & 1.83 & 12.14 & 2.95 & $\mathrm{x}$ & 479.99 & 0.18 & $\mathrm{x}$ & 0 \\
\hline 2 & $b$ & - & - & - & - & - & - & - & - & - & - & - & 0 \\
\hline 3 & $\left(z_{t}-z\right)$ & 1.18 & 70.42 & 1.09 & 1.30 & 1.14 & 12.83 & 5.32 & $\mathrm{x}$ & 37.09 & 1.88 & $\mathrm{x}$ & 0 \\
\hline 4 & $\dot{z}$ & - & - & - & - & - & - & - & - & - & - & - & 0 \\
\hline 5 & $\ddot{z}$ & - & - & - & - & - & - & - & - & - & - & - & 0 \\
\hline 6 & $i, b$ & 1.11 & 105.16 & 0.46 & 0.99 & 4.00 & 29.83 & 2.20 & $\checkmark$ & 0.83 & 0.48 & $\checkmark$ & $0 / 2$ \\
\hline 7 & $b,\left(z_{t}-z\right)$ & 1.18 & 103.09 & 0.41 & 0.95 & 6.74 & 48.60 & 2.56 & $\checkmark$ & 1.94 & 1.26 & $\checkmark$ & $0 / 2$ \\
\hline 8 & $i, b,\left(z_{t}-z\right)$ & 1.67 & 21.52 & 0.43 & 1.31 & 4.88 & 35.84 & 2.11 & $\checkmark$ & 0.60 & 1.48 & $\checkmark$ & $2 / 7$ \\
\hline 9 & $i, b,\left(z_{t}-z\right), \dot{z}$ & 1.69 & 19.13 & 0.35 & 1.28 & 7.18 & 51.57 & 2.19 & $\checkmark$ & 0.21 & 6.05 & $\checkmark$ & $7 / 15$ \\
\hline 10 & $i, b,\left(z_{t}-z\right), \ddot{z}$ & 1.69 & 20.83 & 0.41 & 1.30 & 6.56 & 47.37 & 2.18 & $\checkmark$ & 0.73 & 0.61 & $\checkmark$ & $8 / 15$ \\
\hline 11 & $i, b, \dot{z}, \ddot{z}$ & 1.80 & 18.45 & 0.34 & 1.35 & 7.30 & 52.29 & 2.16 & $\checkmark$ & 0.17 & 8.61 & $\checkmark$ & $6 / 15$ \\
\hline 12 & $b,\left(z_{t}-z\right), \dot{z}, \ddot{z}$ & 1.77 & 19.13 & 0.36 & 1.34 & 6.64 & 47.61 & 2.13 & $\checkmark$ & 0.69 & 6.06 & $\checkmark$ & $5 / 15$ \\
\hline 13 & $i, b,\left(z_{t}-z\right), \dot{z}, \ddot{z}$ & 1.81 & 18.45 & 0.34 & 1.35 & 7.29 & 52.12 & 2.15 & $\checkmark$ & 0.30 & 6.24 & $\checkmark$ & $19 / 31$ \\
\hline
\end{tabular}

$g_{p} \equiv\left(z_{t}-z\right)_{p}, g_{r m s} \equiv\left(z_{t}-z\right)_{r m s}$

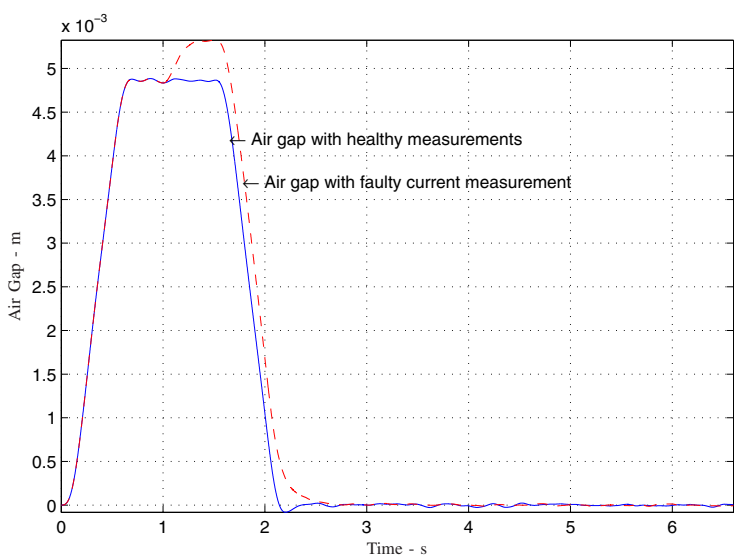

Fig. 7. Air gap deflection with faulty current at $1 \mathrm{sec}$.

\section{B. Future Work}

The proposed sensor optimisation framework is very flexible tool and easily adopted to different sensor optimisation problems. The authors are looking into other complex engineering problems for such purpose.

\section{REFERENCES}

11] M. Blanke, R. Izadi-Zamanabadi, S. A. Bogh, and C. P. Lunau. Fault-tolerant control systems - a holistic view. Control Engineering Practice, 5(5):693-702, 1997.

[2] M. Blanke, M. Kinnaert, J. Lunze, and M. Staroswiecki. Diagnosis and Fault-Tolerant Control. Springer-Verlag New York, Inc., Secaucus, NJ, USA, 2003

[3] C. A. C. Coello. Theoretical and numerical constraint-handling techniques used with evolutionary algorithms: A survey of the state of the art. Computer Methods in Applied Mechanics and Engineering, 191(11-12):1245-1287, 2002.

[4] N. V. Dakev, J. F. Whidborne, A. J. Chipperfield, and P. J. Fleming. Evolutionary $h_{\infty}$ design of an electromagnetic suspension control system for a maglev vehicle. Proceedings of the Institution of Mechanical Engineers.Part I, Journal of Systems \& Control Engineering, 211(5):345-355, 1997.

[5] Kalyanmoy Deb, Amrit Pratap, Sameer Agarwal, and T. Meyarivan. A fast and elitist multiobjective genetic algorithm: Nsga-ii. IEEE Transactions on Evolutionary Computation, 6(2):182-197, 2002.
[6] P. J. Fleming and R. C. Purshouse. Evolutionary algorithms in control systems engineering: A survey. Control Engineering Practice, 10(11):1223-1241, 2002.

[7] B. Friedland. Advanced Control System Design. Prentice-Hall Inc., Upper Saddle River, NJ, USA, 1996.

[8] R. M. Goodall. Dynamic characteristics in the design of maglev suspensions. Proceedings of the Institution of Mechanical Engineers, Part F: Journal of Rail and Rapid Transit, 208(1):33-41, 1994.

[9] R. M. Goodall. Dynamics and control requirements for ems maglev suspensions. In Proceedings on international conference on Maglev, pages 926-934, 2004

[10] R. M. Goodall. Generalised design models for ems maglev. In Proceedings of MAGLEV 2008 - The $20^{\text {th }}$ International Conference on Magnetically Levitated Systems and Linear Drives, 2008.

[11] R. M. Goodall. The theory of electromagnetic levitation. Physics in Technology, 16(5):207-213, Sep 1985.

[12] R. Isermann. Supervision, fault-detection and fault-diagnosis methods-an introduction. Control Engineering Practice, 5(5):639_ 652, 1997.

[13] Abdullah Konak, David W. Coit, and Alice E. Smith. Multi-objective optimization using genetic algorithms: A tutorial. Reliability Engineering and System Safety, 91(9):992-1007, 2006.

[14] K. Michail, A. Zolotas, and R. M. Goodall. Optimised sensor configurations for a maglev suspension. Proceedings of the $17^{\text {th }}$ World Congress The internetional Federation of Automatic Control, pages 8305-8310, July 6-11, 2008.

[15] K. Michail, A. C. Zolotas, R. Goodall, and J. T. Pearson. Sensor optimisation via $h_{\infty}$ applied to a maglev suspension system. International Journal of Computer Systems Science and Engineering, 5(1):43-49, 2009.

[16] K. Michail, A. C. Zolotas, and R. M. Goodall. Ems systems: Optimised sensor configurations for control and sensor fault tolerance. Japan Society of Mechanical Engineers, International Symposium on Speedup, Safety and Service Technology for Railway and Maglev Systems, 2009.

[17] K. Michail, A. C. Zolotas, R. M. Goodall, and J. T. Pearson. Maglev suspensions - a sensor optimisation framework. In $16^{\text {th }}$ Mediterranean Conference on Control and Automation, pages 1514-1519, June 25-27 2008.

[18] R. J. Patton. Fault-tolerant control: The 1997 situation. In IFAC Symposium on Fault Detection Supervision and Safety for Technical Processes, volume 3, pages 1029-1052, 1997.

[19] S. Skogestad and I. Postlethwaite. Multivariable Feedback Control Analysis and Design. John Wiley \& Sons Ltd, $2^{\text {nd }}$ Edition, New York, 2005.

[20] Youmin Zhang and Jin Jiang. Bibliographical review on reconfigurable fault-tolerant control systems. Annual Reviews in Control, 32(2):229252, 122008 . 